\title{
Keynote Lectures
}

[KN10] Thin film solar cells: crystallography from $A$ to $Z$

$\underline{\text { Susan Schorr }}$

\section{Helmholtz Centre Berlin for Materials and Energy, Department Crystallograph, Hahn- Meitner-Platz 1, 14109 Berlin, Germany \\ E-mail: susan.schorr@helmholtz-berlin.de}

The current market dominating photovoltaic devices and modules made from crystalline silicon are $1^{\text {st }}$ generation solar cells. Employing the thin film technology resulted in a significant cost reduction, thus thin film solar cells are considered as $2^{\text {nd }}$ generation solar cells. Thin film photovoltaic modules can processed on large areas and alternative substrates like glass, polyimide foils or stainless steel. Ternary and quaternary compound semiconductors like $\mathrm{CuInSe}_{2}$ (CISe) $\mathrm{Cu}(\mathrm{In}, \mathrm{Ga}) \mathrm{Se}_{2}$ (CIGSe) and $\mathrm{Cu} 2 \mathrm{ZnSn}(\mathrm{S}, \mathrm{Se})_{4}$ (CZTSSe) are candidates for an absorber material in $2^{\text {nd }}$ generation solar cells due to their direct band gap in the range 1-1.5 $\mathrm{eV}$ and high absorption coefficient $\left(>104 \mathrm{~cm}^{-1}\right)$ for solar radiation in the corresponding spectral range. World record device efficiencies reached in the laboratory are $20.3 \%$ for CIGSe based [1] and $11 \%$ for CZTSSe based solar cells [2]. These compounds belong to the group of materials with tetrahedral coordination, i. e. each atom has four nearest neighbours. Such a group of materials is the adamantine compound family, which is based on diamond and lonsdaleite, respectively. Comparing with other semiconductors, for instance Pyrite $\left(\mathrm{FeS}_{2}\right)$ or transition metal chalcogenides (photovoltaic devices with these compounds as absorber layer have shown only low efficiency values), the tetrahedral bonding structure in the adamantine compounds is advantageous in controlling the semiconducing properties of the material. The chalcopyrite type compound CIGSe is extremely tolerant to defects that naturally arise for large deviations from stoichiometry as present in the highestefficiency devices. These deviations, especially
$\mathrm{Cu} /(\mathrm{In}+\mathrm{Ga})<1 \quad$ (copper-poor CIGSe), are balanced by neutral defect complexes like $2 \mathrm{~V}_{\mathrm{Cu}}{ }^{+}$ $\operatorname{In}_{\mathrm{Cu}}$. For the kesterite type compound CZTSSe the homogeneity region is much smaller, i. e. the deviations from stoichiometry are small and secondary phases occur. In order to understand the correlation between growth conditions, material properties and final device quality much has to be understood regarding the basic material properties of the absorber material, for instance the occupation density of the different sites within the crystal structure, i.e. defect formation at certain material compositions. Among various experimental possibilities structural analysis of compound semiconductors by diffraction techniques using X-rays or neutrons has come a key technique of choice. Especially neutron diffraction can provide valuable results, because it allows the differentiation electronical similar elements. The presentation will give an overview of our structural studies [3-7] of off stoichiometric chalcopyrite type and kesterite type compound semiconductors and will demonstrate the advantages of neutron and synchrotron X-ray diffraction.

[1] P. Jackson, et al., Progr. Photovolt.: Res. Appl. 19 (7), 894-897 (2011).

[2] D. Aaron et al., Progr. Photovolt.: Res. Appl. 20 (2012) 6-11.

[3] S. Schorr, Thin Solid Films 515 (15) (2007) 5985-5991.

[4] S. Schorr et al., Europ. Jour. Mineral. 19 (2007) $65-73$.

[5] S. Schorr, Sol. En. Mat. Sol. Cells, 95 (2011) 1482-1488.

[6] C. Stephan et al., Appl Phys Lett 98 (2011) 091906 (091901)-091906 (091903).

[7] C. Stephan, et al., Appl Phys Lett 101 (2012) 101907(101901)-101907(101904).

Keywords: semiconductor defects; crystal structure; neutron diffraction 